\title{
Performance Analysis of Atmospheric Field Conjugation Adaptive Arrays
}

\author{
Aniceto Belmonte*a, Joseph M. Kahn ${ }^{\mathrm{b}}$ \\ a Technical Univ. of Catalonia, Dept. of Signal Theory and Commun., 08034 Barcelona, Spain; \\ ${ }^{\mathrm{b}}$ Stanford University, Department of Electrical Engineering, Stanford, CA 94305, USA \\ *belmonte@tsc.upc.edu
}

\begin{abstract}
System configurations based on single monolithic-apertures that are immune to atmospheric fluctuations are being developed. Main goal is the improvement of the performance achievable in coherent, free-space optical communication systems using atmospheric compensation techniques such as adaptive optics. As an alternative to a single monolithicaperture coherent receiver with a full-size collecting area, a large effective aperture can be achieved by combining the output signal from an array of smaller receivers. We study the communication performance of field conjugation adaptive arrays applied in synchronous laser communication through the turbulent atmosphere. We assume that a single information-bearing signal is transmitted over the atmospheric fading channel, and that the adaptive array coherent receiver combines multiple dependent replicas to improve detection efficiency. We consider the effects of log-normal amplitude fluctuations and Gaussian phase fluctuations, in addition to local oscillator shot noise. We study the effect of various atmospheric parameters and the number of branches combined at the receiver.
\end{abstract}

Keywords: Atmospheric optical communications; Coherent receivers; Diversity combining

\section{INTRODUCTION}

With coherent communications, the complex field of the received signal encodes the transmitted information. To fully measure the complex electrical field, i.e. amplitude and phase, of the light wave, in a coherent detection system the incoming signal interferes with a local oscillator (LO). In a free-space laser receiver the presence of atmospheric turbulence affects the coherence of the received signal that is to be mixed with the local oscillator. Light propagated through a turbulent atmosphere contains speckle which will be present at the detector surface. Therefore, illuminating a single-element detector with a uniform LO beam will produce mismatch of the amplitudes and phases of the two fields resulting in a loss in downconverted power. The downconverted coherent power is maximized when the spatial field of the received signal matches that of the local oscillator. ${ }^{[1],[2]}$

System configurations based on single monolithic-apertures that are immune to atmospheric fluctuations are being developed. ${ }^{[3]}$ Main goal is the improvement of the performance achievable in coherent, free-space optical communication systems using atmospheric compensation techniques. As an alternative to this approach, the performance of diversity combining techniques, where two or more statistically independent fading signals are combined at the receiver, have been considered to improve detection efficiency in coherent laser communication through the turbulent atmosphere. ${ }^{[4][5]}$ Diversity combining consists of receiving redundantly the same information signal over multiple fading channels and to exploit the low probability of concurrence of deep fades in all the diversity channels.

In this analysis, we consider a coherent fiber array consisting of densely packet multiple subapertures, with each subaperture interfaced to a single-mode fiber, as a receiver structure for increasing the performance of the atmospheric laser system. Instead of using a single monolithic-aperture coherent receiver with a full-size collecting area, a large effective aperture can be equally achieved by combining the output signal from a fiber array of smaller subapertures in a close-packed arrangement (see Fig. 1). A coherent fiber array offers an advantage in terms of the coupling efficiency as that the number of turbulence speckles over each subaperture in the array is much smaller than it would be over a single large aperture. Now, each receiver aperture can be smaller than the scale on which the signal wavefront varies and the local oscillator phase can be matched to the signal to attain successful coherent reception. Output signals from these receivers can then be combined electronically to enhance the detection statistics. In general, the performance of such a field conjugation adaptive should improve with an increasing number of subapertures and, given a fixed collecting area,

Free-Space Laser Communication Technologies XXIII, edited by Hamid Hemmati, Proc. of SPIE Vol. 7923, 79230Q · ( 2011 SPIE · CCC code: 0277-786X/11/\$18 · doi: 10.1117/12.873056 
the fiber array system can offer superior performance. Note that, due to the close spatial arrangement of the subapertures in a coherent fiber array, atmospheric fading on the array components is correlated or dependent.

We consider a general statistical model to describe the signal collected by the fiber array receiver after propagation through the atmosphere. We consider the performance of such receivers under the effects of log-normal amplitude fluctuations and Gaussian phase fluctuations, in addition to local oscillator shot noise.

\section{A STATISTICAL MODEL FOR THE RECEIVER}

In a coherent communication receiver, the SNR $\gamma_{0}$ per unit bandwidth $B$ for a quantum or shot-noise limited signal can be interpreted as the detected number of photons (photocounts) per symbol when $1 / B$ is the symbol period. Coherently detected signals are modeled as narrowband RF signals with additive white Gaussian noise (AWGN). For a coherent receiver system, in the presence of target speckle and atmospheric turbulence, we must consider fading signals, which are signals also affected by multiplicative noise. In the fading AWGN channel, we let $\alpha^{2}$ denote the atmospheric channel power fading and $\gamma_{0} \alpha^{2}$ denote the instantaneous received SNR per pulse. For a shot-noise-limited coherent optical receiver, the SNR of the envelope detector can be taken as the number of signal photons detected on the receiver aperture $\gamma_{0}$ multiplied by a heterodyne power mixing efficiency $\alpha^{2}$. For systems with perfect spatial mode matching, the mixing efficiency is equal to 1 . When the spatial modes are not properly matched, the contribution to the current signal from different parts of the receiver aperture can interfere destructively and result in the reduced instantaneous heterodyne mixing and consequent fading. Note that, conditional on a realization of the atmospheric channel described by $\alpha^{2}$, this is an AWGN channel with instantaneous received SNR $\gamma=\gamma_{0} \alpha^{2}$. This quantity is a function of the random channel power fading $\alpha^{2}$, and is therefore random. The statistical properties of the atmospheric random channel fade $\alpha$, with probability density function (PDF) $p_{\alpha}(\alpha)$, provide a statistical characterization of the SNR $\gamma=\gamma_{0} \alpha^{2}$. In this study we define a statistical model for the fading amplitude $\alpha$ (i.e., SNR $\gamma$ ) of the received signal after propagation through the atmosphere.

In a single-aperture, fiber-based coherent receiver, when the spatial field of the received signal $E_{i}(\mathbf{r})$ does not match that of the local oscillator $E_{m}(\mathbf{r})$, as described by the fiber-mode profile referred to the receiving aperture, the random fading

$$
\alpha=\frac{4}{\pi D^{2}} \int d \mathbf{r} W(\mathbf{r}) E_{i}(\mathbf{r}) E_{m}(\mathbf{r})
$$

depends in amplitude and phase mismatches of the two fields incident on the receiving aperture. Phase and amplitude mismatches represent the amplitude fluctuations and phase distortions introduced by atmospheric turbulence in the received signal. The circular receiving aperture of diameter $D$ is defined by the aperture function $W(\mathbf{r})$, which equals unity for $|\mathbf{r}| \leq D / 2$, and equals zero for $|\mathbf{r}|>D / 2$.

In general, in Eq. (1) fading is a complex magnitude $\alpha=\alpha_{r}+j \alpha_{i}$ where $\alpha_{r}$ and $\alpha_{i}$ represent integrals over the collecting aperture of the real and imaginary parts, respectively, of the optical fields reaching the receiver. These real and imaginary parts can be considered as the components of a complex random phasor. We need to study how amplitude and phase turbulence-induced fluctuations of the optical field define the statistics of the fading intensity $\alpha=\alpha_{r}+j \alpha_{i}$. From Eq. (1), we note that the two random magnitudes $\alpha_{r}$ and $\alpha_{i}$ can be expressed as integrals over the aperture and, hence, are the sums of contributions from each point in the aperture. In order to proceed with the analysis, we could consider a statistical model in which these continuous integrals are expressed as finite sums over statistically independent cells in the aperture. Under the assumption that the number of independent coherent regions is large enough, we can consider that $\alpha_{r}$ and $\alpha_{i}$ asymptotically approach jointly normal random variables. Then, the probability density function of the length fading amplitude $\alpha$ can be well approximate by a Rayleigh distribution. Just as in a speckle pattern, the Rayleigh distribution for the turbulence amplitude fading length is a consequence of the central-limit theorem. However, under conditions of weak-turbulence in which the number of coherent terms is small, the fading may actually be the result of summing a small number of terms. In this case, the fading $\alpha$ is not likely to be Rayleigh. Rather than assuming that $\alpha$ is always Rayleigh distributed for all conditions of turbulence, it is more realistic to assume that $\alpha$ satisfied a generalized Rayleigh distribution that becomes Rayleigh only when the number of coherent terms $N$ becomes large enough. Such a distribution is the Nakagami-m distribution, ${ }^{[6]}$ in essence a central chi-square distribution described by: 


$$
p_{\alpha}(\alpha)=2(m N)^{m} \frac{\alpha^{2 m-1}}{\Gamma(m)} \exp \left(-m N \alpha^{2}\right)
$$

where $\Gamma$ is the complete gamma function. The Nakagami-m parameter $m$ and fading parameter $N$ are a measure of turbulence effects. Here, $N$ is the inverse of the fading mean-square value

$$
N=1 / \overline{\alpha^{2}} \text {. }
$$

The parameter $m$ characterizes the amount of turbulent fading. When $m \rightarrow 1$, the number of contribution coherent areas $N$ is large and the m-distribution reduces to Rayleigh. Note that the Nakagami-m distribution closely approximates the Rice distribution ${ }^{[6]}$ we have previously used to model the impact of atmospheric turbulence-induced fading on free-space optical communication links using coherent detection. ${ }^{[3]}$ Applying the Jacobian of the transformation $\alpha^{2}=\gamma / \gamma_{0}$, the corresponding SNR $\gamma$ distribution can be described according to a gamma distribution with a shape parameter $m$ and a mean value $\bar{\gamma}=\gamma_{0} \overline{\alpha^{2}} \equiv \gamma_{0} / N$ given by

$$
p_{\gamma}(\gamma)=\left(\frac{m N}{\gamma_{0}}\right)^{m} \frac{\gamma^{m-1}}{\Gamma(m)} \exp \left(-\frac{m N}{\gamma_{0}} \gamma\right) .
$$

When $m \rightarrow 1$, the gamma distribution reduces to exponential distribution. Note that the PDF in Eq. (4) can equivalently be expressed in terms of its moment generating function (MGF), which it is closely related to the Laplace transform of the distribution $p_{\gamma}(\gamma)$ and defined as the expected value of $\exp (s \gamma)$ :

$$
\begin{aligned}
M_{\gamma}(s) & =\int_{0}^{\infty} d \gamma \exp (s \gamma) p_{\gamma}(\gamma) \\
& =\left(1-s \frac{\gamma_{0}}{m N}\right)^{-m}=\left(1-s \frac{\gamma_{0}}{m} \overline{\alpha^{2}}\right)^{-m} .
\end{aligned}
$$

The MGF is a useful tool for analyzing the average error probability in communication systems with fading. Also, it can be shown that the distribution moments are given by

$$
\overline{\gamma^{k}}=\frac{\Gamma(m+k)}{m^{k} \Gamma(m)} \bar{\gamma}^{k},
$$

which yields, by using the average SNR, an expression for $m$

$$
\frac{1}{m}=\frac{\overline{\sigma_{\gamma}^{2}}}{\bar{\gamma}^{2}}=\frac{\overline{\alpha^{4}}}{{\overline{\alpha^{2}}}^{2}}-1 .
$$

Since $\alpha_{r}$ and $\alpha_{i}$ can be considered jointly normal random variables, it is possible to relate high-order moments with the lower-order moments and replaces the fourth-order moment in Eq. (7) by $\overline{\alpha^{4}}=3{\overline{\alpha^{2}}}^{2}-2 \bar{\alpha}^{4}$. It results:

$$
\frac{1}{m}=2-2\left(\frac{\bar{\alpha}^{2}}{\overline{\alpha^{2}}}\right)^{2} .
$$

The parameter $m$, by characterizing the amount of fading through the normalized SNR $\gamma$ variance, gives more control over the extent of the turbulence fading. When $m \rightarrow 1$ and the number of contribution coherent areas is large, the normalized variance is one, as expected for Rayleigh distributions. When $m$ grows $m \rightarrow \infty$, and a very small number of contribution terms add together, the normalized variance decreases. Now, the density function becomes highly peaked around the mean value $\bar{\gamma}=\gamma_{0} / N$ and there is just a small fading to be considered.

To have a measure of turbulence effects, it has been necessary to develop procedures to estimate $m$ and $N$. Equivalently, as Eqs. (3) and (8) describe fading parameters $m$ and $N$ in terms of fading first and second moments, we have needed to 
establish closed expressions for $\bar{\alpha}$ and $\overline{\alpha^{2}}$. In order to assess the impact of turbulence on the heterodyne mixing and fading, the field amplitude without the effect of turbulence in the pupil plane must be modified by a multiplicative factor $\exp [\chi(\boldsymbol{r})-j \phi(\boldsymbol{r})]$ where $\chi(\boldsymbol{r})$ and $\phi(\boldsymbol{r})$ represent the log-amplitude fluctuations (scintillation) and phase variations (aberrations), respectively, introduced by atmospheric turbulence. Consequently, both $\bar{\gamma}$ and $1 / r$ are described in terms of log-normal amplitude fluctuations and Gaussian phase fluctuations as characterized by their respective statistical variances, $\sigma_{\chi}^{2}$ and $\sigma_{\phi}^{2}$. Also, the propagating mode of a single-mode fiber is well approximated by an untruncated Gaussian function and the fiber-mode profile referred to the receiving aperture describing the local oscillator $E_{m}(\mathbf{r})$ can be characterized by its fiber-mode field radius at the front surface of the receiving lens $\omega_{m}$.

We define a general model for the output SNR of diversity systems over correlated fading channels. For field conjugation adaptive arrays, where the atmospheric fading on the branches is correlated or dependent, we can solve the problem by transforming it into an independent problem using the technique of spatial whitening. At this way, the results for independent fading channels are easily extended to the more general problem of correlated channels.

\section{PERFORMANCE OF A CLOSELY PACKED HEXAGONAL COHERENT ARRAY}

In a field conjugation fiber array, the intermediate-frequency signals at the output of the single-mode fibers needs to be co-phased and their amplitude independently adapted before they are summed to lessen signal fading associated with atmospheric turbulence and reduce fiber coupling shortcomings. Note that this is equivalent to consider maximum ratio combining (MRC) of the received signals by the array subapertures as they can be considered as branches of a diversity combiner. ${ }^{[7]}$ MRC diversity schemes assume perfect knowledge of the branch amplitudes and phases, require independent processing of each branch, and need that the individual signals from each branch be weighted by their signal to noise power ratios then summed coherently. A receiver with MRC will coherently combine the diversity branches by weighting them by the complex conjugate of their respective fading gains and adding them. Clearly, like in a MRC combiner, the instantaneous SNR $\gamma_{T}$ for a summing coherent array is the power ratio of the phase-coherent addition of the signal amplitudes from each element of the array to the incoherent addition of the noise. If an optimum voltage gain proportional to the amplitude of the signal itself is assumed for each element in the array, and if equal noise powers are assumed, the resultant composite SNR $\gamma_{T}$ for an $L$-element coherent fiber array is the sum of the array elements $\operatorname{SNR} \gamma_{l}$. For independent subaperture signals and equal average branch SNR, i.e. $\bar{\gamma}_{l}=\bar{\gamma}$ for all $l \quad\{1,2, \ldots, L\}$, the PDF of the received SNR $\gamma_{T}$ at the output of a perfect $L$-branch coherent array in the atmosphere would be described a sum of $L$ independent and identically distributed gamma random variables. This random variable it is also described by the gamma distribution Eq. (4) with a shape parameter $(m L)$. However, for close coherent fiber arrays receivers, with insufficient collecting apertures spacing, it is not realistic to assume that the combined signals are independent of one another. In this scenario, the degree of correlation among the different fadings $\alpha_{l}$ describing $\gamma_{T}$ will depend on several factors, including atmospheric conditions and the exact geometry of the coherent array receiver.

It is worthy of mentioning that the evaluation of the most common performance measures of an optical coherent communication system in the presence of atmospheric fading can be accomplished based entirely on the knowledge of the MGF of the output SNR without ever having to compute its PDF. We will use $M_{T}(s)$ to estimate the performance of the MRC coherent fiber array. This MGF-based approach is quite useful in simplifying our analysis.

Figures 3 and 4 consider the mean and standard deviation of the SNR at the output of field conjugation adaptive arrays. We contemplate an $L$-element coherent fiber array and assume that the subapertures are arranged in a hexagonal closepacked array as shown in Fig. 2. The larger circle represents a single receiver aperture of diameter $D$. The small circles represent packed subapertures and each subaperture contains a lens that couples the received light into a single-mode fiber. For comparison of the receiver performance between an L-element coherent array and a single large aperture, we force the hexagonal array to be packed within the limits of the single aperture area. The hexagonal distribution is the densest way to arrange circles in a plane. Still, note that each subaperture will have a pupil area slightly smaller than $1 / L$ times the pupil area of the single receiver system and an array fill factor needs to be considered in our analysis. For instances, for the array with $L=7$ elements in Fig. 1, the array fill factor is $7 / 9$. Also, the truncation parameter of the pupil $\tau \equiv D / 2 \omega_{m}$ describes the ratio of the receiver aperture diameter to the diameter of the backpropagated fiber mode. A large 
value of $\tau$ or $D \square 2 \omega_{m}$ represents a narrow Gaussian mode or a weakly truncated pupil. A uniform illuminated pupil is obtained by letting $\tau \rightarrow 0$. The truncation parameter must be chosen to optimize the receiving system performance. Although the optimum parameter may depend on the level of atmospheric turbulence considered, this dependency is very weak and we can chose the optimum value of 1.12 obtained in absence of turbulence, when the incident plane wave is fully coherent. ${ }^{[8]}$

We study the mean SNR in Fig. 3 and the SNR normalized standard deviation in Fig. 4 as a function of several parameters: the average turbulence-free SNR $\gamma_{0}$, the receiver aperture diameter $D$, the number of subapertures $L$ of the hexagonal distributed fiber array coherently summed, and the strength of atmospheric turbulence. Turbulence is quantified by two parameters: the phase coherence length $r_{0}$, which describes the spatial correlation of phase fluctuations in the receiver plane, ${ }^{[1]}$ and the scintillation index $\sigma_{\beta}{ }^{2} \cdot{ }^{[9]}$ The value of the scintillation index $\sigma_{\beta}{ }^{2}=1$ corresponds to strong scintillation, but still below the saturation regime. When we assume no scintillation, $\sigma_{\beta}^{2}=0$, the effect of turbulence is simply to reduce the coherence length $r_{0}$. For a fixed coherent diameter $r_{0}$, as aperture diameter $D$ is increased, the normalized aperture diameter $D / r_{0}$ increases, and turbulence reduces the heterodyne downconversion efficiency.

In Fig. 3, the mean SNR is plotted against the normalized aperture diameter $D / r_{0}$ for different number of subapertures $L$ on the hexagonal array. The SNR is expressed in $\mathrm{dB}$, referenced to the turbulence free SNR $\gamma_{0}$. This corresponds to the mean intensity fading $\overline{\alpha^{2}}=1 / N$ according to the transformation $\gamma=\gamma_{0} \alpha^{2}$. Note that the mean SNR is just a representation of the fiber-coupling efficiency. The received signal beam must be coupled into a single-mode fiber but atmospheric turbulence degrades the spatial coherence of a laser beam and limits the fiber-coupling efficiency and, consequently, the available mean SNR at the output of each fiber in the array. As expected for one single monolithic aperture $L=1$, if $\mathrm{D}$ is less than $r_{0}$, the normalized mean SNR $\bar{\gamma} / \gamma_{0}$ remains constant. A truncation parameter $\tau=1.12$ reduces the mean SNR by $5 \mathrm{~dB}$. When diameter $\mathrm{D}$ is larger than $r_{0}$, atmospheric turbulence limits the effective receiving aperture to the dimensions of the coherence diameter $r_{0}$ and the normalized mean SNR goes down very quickly. When the normalized aperture diameter $D / r_{0}$ is large, an increase in the number of the hexagonal array subapertures improves the situation significantly. For instances, when a large normalized aperture $D / r_{0}=10$ is considered, increasing the number of subapertures $L$ from 1 to 19 commends the mean SNR by more than $13 \mathrm{~dB}$. The mean SNR is just a representation of the fiber-coupling efficiency: the advantage of a fiber array in terms of the mean SNR and the fiber coupling efficiency is that the number of field coherence areas $N$ over each subaperture is smaller than it would be over a single large aperture.

In Fig. 4, we plot the normalized SNR standard deviation (SNR uncertainty or relative error) $\sigma_{\gamma} / \bar{\gamma}$ against the normalized aperture diameter $D / r_{0}$ for different values of the number of subapertures $L$ on the hexagonal array. In the limit of weak turbulence (small normalized aperture diameter $D / r_{0}$ ), the normalized variance trends asymptotically to 0 . When aperture diameter is increased, SNR uncertainty grows steadily with the number of field coherence areas $N$ over the receiving pupil until reaching a well define maxim. In the limit of strong turbulence (large normalized aperture diameter $D / r_{0}$ ), the SNR uncertainty becomes flat and very intense. When $L=1$, the SNR standard deviation reach a maximum value of almost $1.6(2 \mathrm{~dB})$. Once again, an increase in the number of the array subapertures will improve the situation and decrease SNR uncertainty: As an example, when the normalized aperture $D / r_{0}=10$ is considered, increasing the number of subapertures $L$ from 1 to 19 decrease the mean SNR by roughly $6 \mathrm{~dB}$, from $1 \mathrm{~dB}$ to $-5 \mathrm{~dB}$. As we observe in Fig. 4, the effects of scintillation are noticeable for the small aperture diameters and must be properly considered. For relatively small apertures, amplitude scintillation is dominant, and normalized variance is virtually unaffected by wavefront phase distortions. When the aperture is larger, phase distortion becomes dominant and the scintillation index $\sigma_{\beta}{ }^{2}$ is of little relevance in the SNR uncertainty. Also, it results of interest to consider the case of independent subapertures. These independent signals could be obtained by spacing the multiple subapertures in the array. If the output of a perfect $L$-elements combiner in the atmosphere would be described as a sum of $L$ independently fading signals, the output SNR uncertainty would be apparently better. That is the case in the limit of weak turbulence or small normalized apertures, where, for instances, when 7-elements arrays are considered, the uncertainty is almost $4 \mathrm{~dB}$ smaller for ideal independent subapertures. This 4-dB difference is the penalty for using closely packed arrays that combine highly correlated signals. However, when strong turbulence or large normalized apertures are considered, field coherence areas are smaller than any array subaperture used in this analysis and the signals collected by then are mostly uncorrelated. In this regime, the multiple replicas combined at the array receiver are statistically independent and to distance the elements of the array would not improve the SNR uncertainty. 
Figures 5 and 6 presents the $\mathrm{SEP}^{[10][11]}$ for an $L$-elements coherent fiber array receiver. Figure. 5 shows the performance SEP vs. normalized aperture diameter $D / r_{0}$, while Fig. 6 shows the performance vs. turbulence-free SNR $\gamma_{0}$. In Fig. 5, for the smallest aperture diameter considered, the turbulence-free SNR has a value $\gamma_{0}=0 \mathrm{~dB}$. For any other aperture diameter, the value of $\gamma_{0}$ is proportional to $D^{2}$. When we assume no scintillation, $\sigma_{\beta}{ }^{2}=0$, the effect of turbulence is simply to reduce the coherence length $r_{0}$. For a single large aperture, even using a relatively small normalized aperture diameter $D / r_{0}=1$, turbulence introduces a strong performance penalty at SEP. When multiaperture array receivers are considered, in most situations it yields a substantial performance improvement. An array with just $L=19$ subapertures yields significant improvement for even the largest normalized apertures considered. The performance of such array receiver is very close to the performance expected in an AWGN system. In Fig. 5, when large normalized apertures $D / r_{0}$ are considered, the SEP becomes independent of the scintillation index $\sigma_{\beta}{ }^{2}$, and tends toward an asymptotic value that is independent of normalized aperture diameter $D / r_{0}$.

Figure 6 shows the performance for different values of $L$, the number of array elements. Even using a relatively small normalized aperture diameter $D / r_{0}=2$, when a single large aperture receiver is used, turbulence introduces more than a 30-dB performance penalty at $10^{3}$ SEP. When multielement arrays are used, performance improves markedly. For instances, considering a small array receiver with $L=7$ subapertures, at a SEP $=10^{-3}$ the SNR penalty is just below $10 \mathrm{~dB}$. This value should be contrasted with the 6-dB penalty observed in Fig. 6 when $L=7$ independently fading signals are ideally combined. Also, although this results assume no scintillation, when we impose a strong scintillation index of $\sigma_{\beta}^{2}=1$ the penalty increase is less than $1 \mathrm{~dB}$ at $\mathrm{SEP}=10^{-3}$.

\section{CONCLUSIONS}

We have numerically evaluated the performance of adaptive field conjugation array receivers in coherent laser communications through the turbulent atmosphere. We analyze coherent fiber arrays consisting of densely packet multiple subapertures in a hexagonal arrangement and consider the effects of log-normal amplitude fluctuations and Gaussian phase fluctuations, in addition to local oscillator shot noise. By noting that the impact of atmospheric turbulence on coherent receivers can be statistically described by a Nakagami-m probability density function, our model uses fundamental principles of atmospheric propagation and circumvents the need for a detailed description of the turbulence problem.

For fiber adaptive arrays, where the atmospheric fading on the subapertures is correlated or dependent, we can solve the problem by transforming it into an independent problem. A MGF-based approach used in our analysis has provided easily evaluable analytical expressions for the signal statistical moments and the symbol error probabilities. We have used it to study the effect of various parameters on performance, including turbulence level, signal strength, receive aperture size, and the number of subapertures in the coherent fiber array. We have separately quantified the effects of amplitude fluctuations and wavefront phase distortion on system performance, and have identified different regimes of turbulence. For most typical free-space laser communication situations, using coherent arrays with a reasonably small number of subapertures such as $L=19$ increases the communication performance by several decibels.

The research of Aniceto Belmonte was partially funded by the Spanish Department of Science and Innovation MICINN Grant No. TEC 2009-10025.

\section{REFERENCES}

[1] D. L. Fried, "Optical heterodyne detection of an atmospherically distorted signal wave front," Proc. IEEE 55, 57-67 (1967).

[2] R. M. Gagliardi and S. Karp, Optical Communications (John Wiley \& Sons, 1995).

[3] A. Belmonte and J. M. Kahn, "Performance of synchronous optical receivers using atmospheric compensation techniques," Opt. Express 16, 14151-14162 (2008).

[4] E. J. Lee and V. W. Chan, "Diversity Coherent and Incoherent Receivers for Free-Space Optical Communication in the Presence and Absence of Interference," J. Opt. Commun. Netw. 1, 463-483 (2009).

[5] A. Belmonte and J. M. Kahn, "Capacity of coherent free-space optical links using diversity-combining techniques," Opt. Express 17, 12601-12611 (2009). 
[6] M. Nakagami, "The m-distribution. A general formula of intensity distribution of rapid fading," in Statistical Methods in Radio Wave Propagation, W. C. Hoffman, ed. (Pergamon Press, 1960).

[7] J. D. Parsons, "Diversity techniques in communications receivers," in Advanced Signal Processing, D. A. Creasey, ed. (Peregrinus, 1985), Chap. 6.

[8] P. J. Winzer and W. R. Leeb, "Fiber coupling efficiency for random light and its applications to lidar," Opt. Letters 23, 986-988 (1998).

[9] J. W. Strohbehn, T. Wang, and J. P. Speck, "On the probability distribution of line-of-sight fluctuations of optical signals," Radio Science 10, 59-70 (1975).

[10] J. G. Proakis and M. Salehi, Digital Communications, (Mc Graw-Hill, 2007).

[11] M. K. Simon and M.-S. Alouini, "A unified approach to the performance analysis of digital communications over generalized fading channels,” IEEE Proc. 86, 1860-1877 (1998).

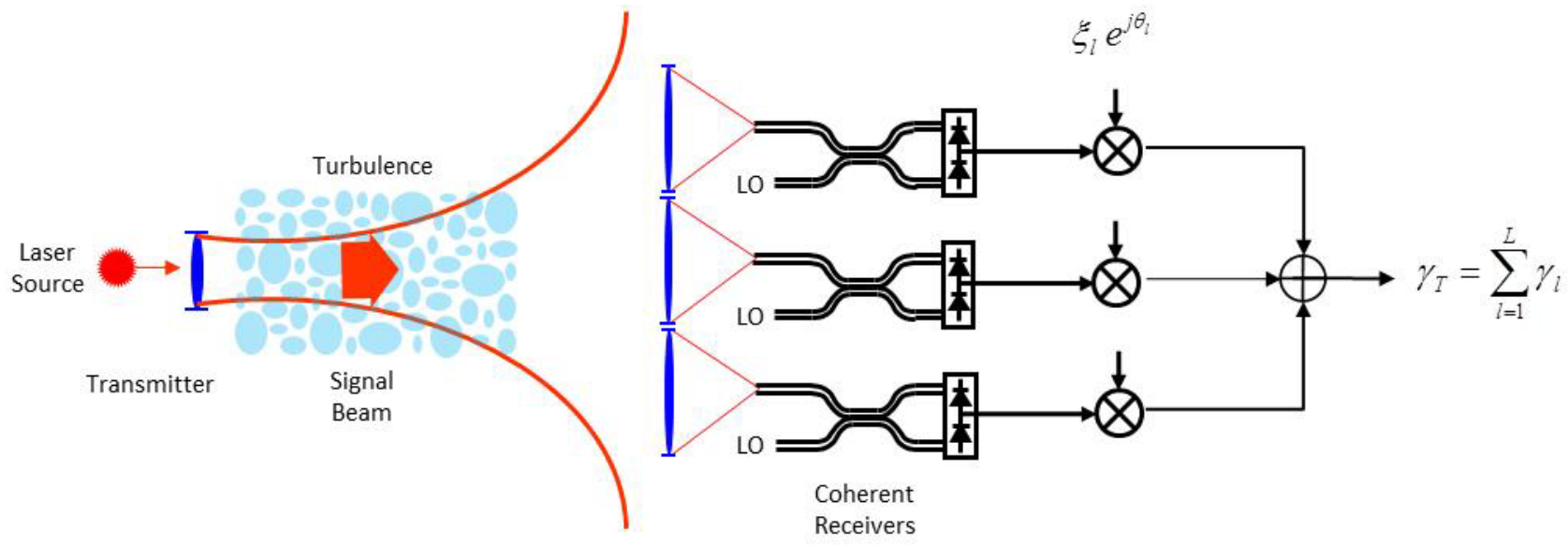

Figure 1. A coherent free-space optical communication system is affected by the presence of atmospheric turbulence in many ways. Amplitude scintillation and phase distortion in the receiver plane act as intense sources of noise distorting the quality of the optical signal available for processing and add together to deteriorate the overall communication performance of the optical systems. In a field conjugation fiber array, the intermediate-frequency signals at the output of the single-mode fibers are adaptively co-phased, have their amplitude separately adjusted, and then summed to mitigate signal fading associated with atmospheric turbulence.

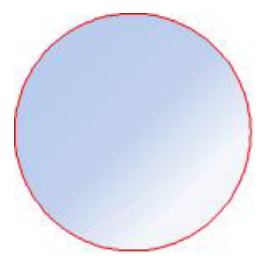

$\mathrm{L}=1$

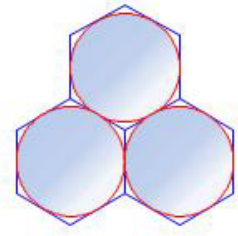

$\mathrm{L}=3$

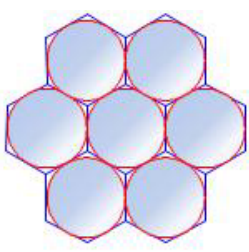

$\mathrm{L}=7$

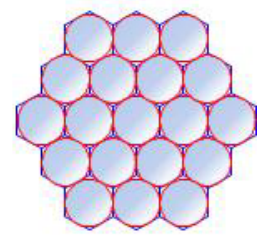

$\mathrm{L}=19$

Figure 2. Adaptive arrays could be considered to alleviate the deteriorating effects of atmospheric turbulence. We consider $L$ element coherent fiber arrays and assume that the subapertures are arranged in a hexagonal close-packed array. 


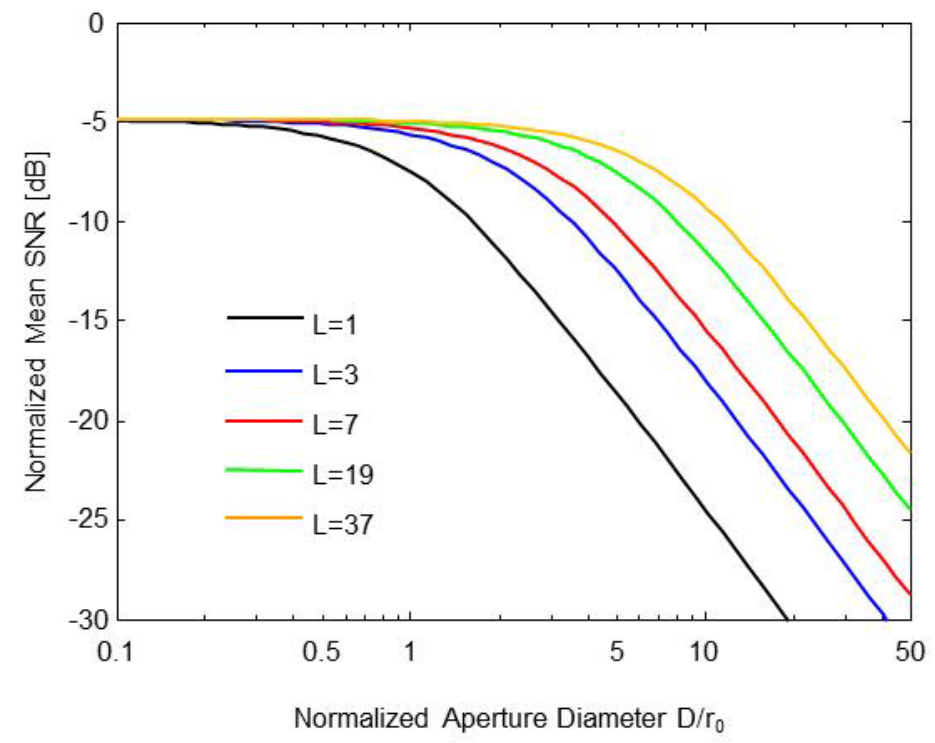

Figure 3. Normalized mean coherent SNR vs. the normalized receiver aperture diameter $D / r_{0}$. Performance is shown for different values of the number of of supapertures $L$ in the closely packed hexagonal coherent array. The case $L=1$ corresponds to a monolitic aperture (black line). When a single aperture is considered, $D$ describes the receiver aperture diameter. For comparison of the receiver performance between an L-element coherent array and a single large aperture, we force the hexagonal array to be packed within the limits of the single aperture diameter $D$. The analysis contemplates the array fill factor and considers a coupling-geometry parameter $\tau$ for each subaperture lens equal to 1.12 .

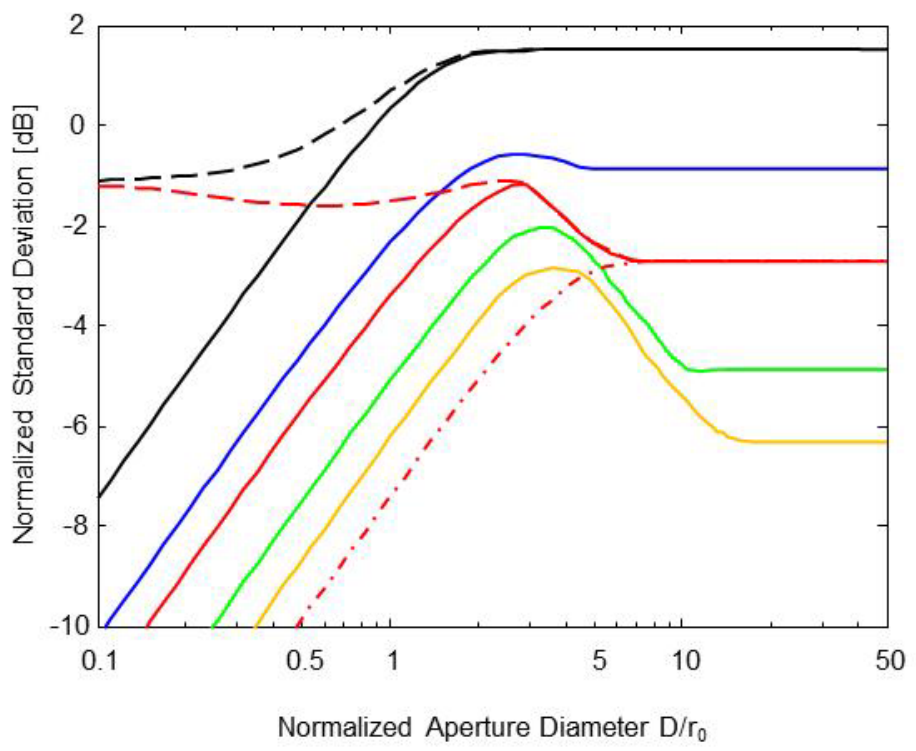

Figure 4. SNR normalized standard deviation vs. the normalized receiver aperture diameter $D / r_{0}$. Performance is shown for different values of the number of of supapertures $L$ in the closely packed hexagonal coherent array. The case $L=1$ corresponds to a monolitic aperture (black line). When a single aperture is considered, $D$ describes the receiver aperture diameter. For comparison of the receiver performance between an L-element coherent array and a single large aperture, we force the hexagonal array to be packed within the limits of the single aperture diameter $D$. The analysis contemplates the array fill factor and considers a coupling-geometry parameter $\tau$ for each subaperture lens equal to 1.12.. Amplitude fluctuations are neglected (solid line) by assuming $\sigma_{\beta}{ }^{2}=0$. When scintillation is considered (dashed line), the scintillation index is fixed at $\sigma_{\beta}{ }^{2}=1$. The red, dotted line corresponds to the uncertainty associated with $L=7$ independent-subapertures limit where fading correlation is neglected. 


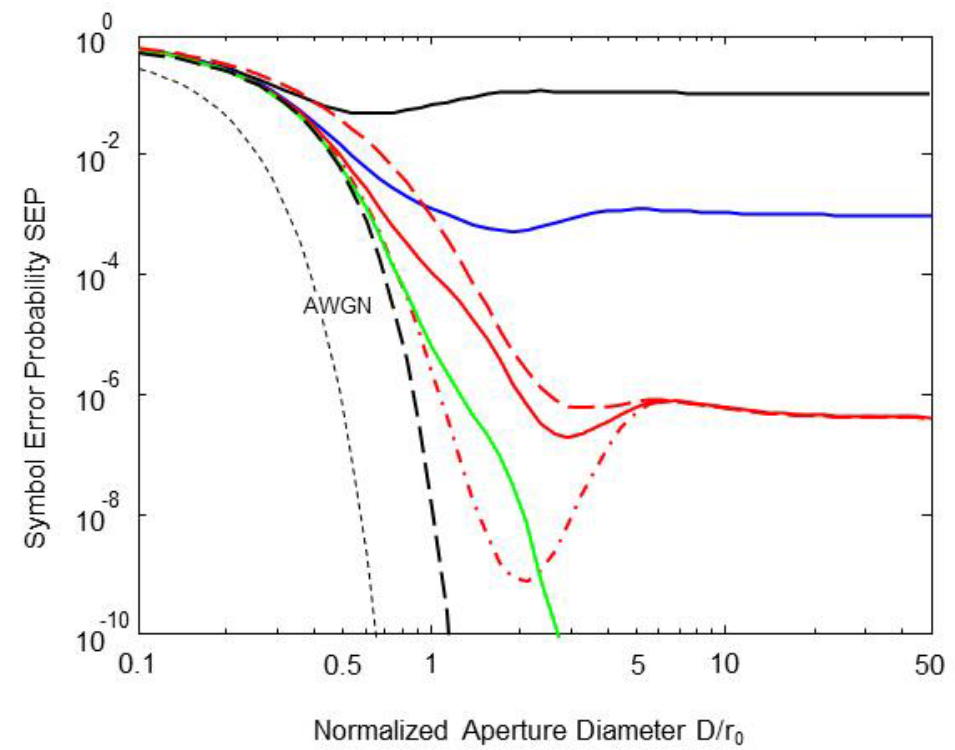

Figure 5. SEP vs. normalized receiver aperture diameter $D / r_{0}$ for QPSK with coherent detection and AWGN. Performance is shown for different values of the number of of supapertures $L$ in the closedly packed hexagonal coherent array. The case $L=1$ corresponds to a monolitic aperture (black line). When a single aperture is considered, $D$ describes the receiver aperture diameter. For comparison of the receiver performance between an L-element coherent array and a single large aperture, we force the hexagonal array to be packed within the limits of the single aperture diameter $D$. The analysis contemplates the array fill factor and considers a coupling-geometry parameter $\tau$ for each subaperture lens equal to 1.12. The turbulence-free SNR per symbol $\gamma_{0}$ is proportional to the square of the aperture diameter $D$. For the smallest aperture considered, we assume $\gamma_{0}=0 \mathrm{~dB}$. In all cases, amplitude fluctuations are neglected (solid line) by assuming $\sigma_{\beta}{ }^{2}=0$. When scintillation is considered for $L=7$ (red, dashed line), the scintillation index is fixed at $\sigma_{\beta}{ }^{2}=1$. Also for $L=7$, the red, dotted line corresponds to the SEP associated with the independent-subapertures limit where fading correlation is neglected.The no-turbulence, AWGN case is indicated by black, dashed lines. The best performance shown inthis plots (dot black line) considers the AWGN limit with $\tau=0$ to obtain a uniform illuminated pupil. When compared with the $\tau=1.12$ case, the mean SNR is now 5-dB higher.

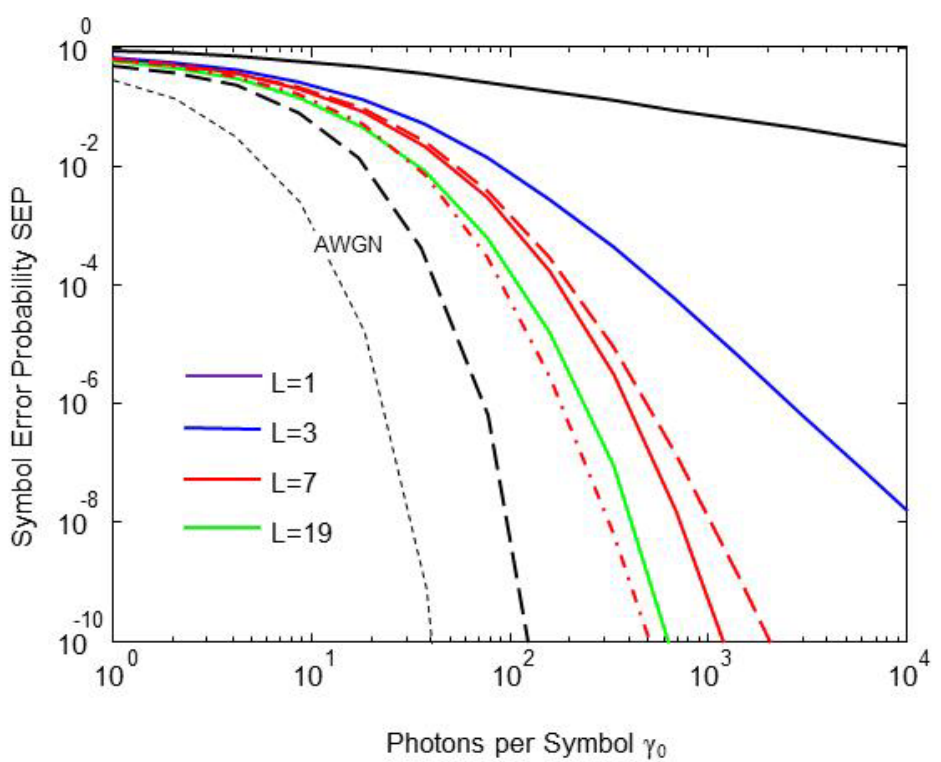

Figure 6. SEP vs. number of photons per symbol for QPSK with coherent detection and AWGN. Performance is shown for different values of the number of of supapertures $L$ in the closedly packed hexagonal coherent array. The normalized aperture diameter $D / r_{0}$ is set to 2. Other parameters are similar to those in Fig. 5. Once again, amplitude fluctuations are neglected (solid line) by assuming $\sigma_{\beta}{ }^{2}=0$. When scintillation is considered for $L=7$ (red, dashed line), the scintillation index is fixed at $\sigma_{\beta}{ }^{2}=1$. Also for $L=7$, the red, dotted line corresponds to the SEP associated with the independent-subapertures limit where fading correlation is neglected. 